\title{
Interference Alignment and the Generalized Degrees of Freedom of the $X$ Channel
}

\author{
Chiachi Huang, Viveck R. Cadambe, Syed A. Jafar \\ Electrical Engineering and Computer Science \\ University of California Irvine \\ Irvine, California, USA \\ Email: $\{$ chiachih, vcadambe, syed $\} @$ uci.edu
}

\begin{abstract}
We study the sum capacity of the $X$ channel generalization of the symmetric 2-user interference channel. In this $X$ channel, there are 4 independent messages, one from each transmitter to each receiver. We characterize the sum capacity of a deterministic version of this channel, and obtain the generalized degrees of freedom characterization for the Gaussian version. The regime where the $X$ channel outperforms the underlying interference channel is explicitly identified, and an interesting interference alignment scheme based on a cyclic decomposition of the signal space is shown to be optimal in this regime.
\end{abstract}

\section{INTRODUCTION}

The $X$ channel is a generalization of the 2-user interference channel with 4 independent messages, one from each transmitter to each receiver. It holds special significance for a number of reasons:

1) Interference Alignment: It is the smallest (in terms of the number of nodes) network where the newly discovered concept of interference alignment [1], [2] becomes relevant.

2) Structured Codes: The $X$ channel is related to the "double dirty" multiple access channel [3] where linear codes have been shown to outperform random codes. This is because, like the "double dirty" multiple access channel, each transmitter in an $X$ channel has side-information about its own potentially interfering transmissions to the other receiver.

In this paper, we explore the capacity of the $X$ channel as a stepping stone to an improved understanding of the role of interference alignment and structured coding in wireless networks. For simplicity of exposition, we choose as a baseline the symmetric interference channel model and study its $X$ channel extension. We identify the regime where interference alignment is helpful so that the $X$ channel has a higher capacity than the underlying symmetric interference channel. The interference alignment is accomplished using a linear coding scheme based on a cyclic decomposition of the signal space which is of interest in and of itself.

Etkin, Tse, and Wang [4] introduced the notion of generalized degrees of freedom (GDOF) to study the performance of various interference management schemes. As its name suggests, the idea of GDOF is a generalization of the concept of degrees of freedom. Unlike the conventional degrees of freedom perspective where all signals are approximately equally strong in the $d B$ scale, the GDOF perspective provides a richer characterization by allowing the full range of relative signal strengths in the $d B$ scale.

A useful technique in the characterization of the GDOF of a wireless network is the deterministic approach [5]. The deterministic approach essentially maps a Gaussian network to a deterministic channel, i.e, a channel whose outputs are deterministic functions of its inputs. The deterministic channel captures the essential structure of the Gaussian channel, but is significantly simpler to analyze. Reference [6] showed that the deterministic approach leads to a GDOF characterization of the 2-user interference network, which leads to a constant bit approximation of its capacity.

In this paper, using a deterministic approach, we characterize the sum capacity of a deterministic $X$ channel, and obtain the GDOF characterization for the Gaussian version. Although the models are simplified to symmetric setting, our results provide an interesting view of the structural difference between the $X$ channel and the interference channel. In terms of GDOF, both channels perform equally well when interference alignment is not applicable. But when interference alignment is applicable, the $X$ channel has larger GDOF and, therefore, higher capacity than the interference channel.

The rest of the paper is organized as follows. Section II describes the models. Section III summarizes our main results. In Section IV, we explore the sum capacity of the deterministic $X$ channel. Section $\mathrm{V}$ provides the GDOF characterization of the symmetric Gaussian $X$ channel.

\section{SySTEM MODEL}

\section{A. The Symmetric Deterministic X Channel}

The symmetric deterministic $X$ channel is described by the input-output equations

$$
\begin{aligned}
\mathbf{Y}_{1}(t) & =\mathbf{S}^{q-n_{d}} \mathbf{X}_{1}(t)+\mathbf{S}^{q-n_{c}} \mathbf{X}_{2}(t) \\
\mathbf{Y}_{2}(t) & =\mathbf{S}^{q-n_{c}} \mathbf{X}_{1}(t)+\mathbf{S}^{q-n_{d}} \mathbf{X}_{2}(t)
\end{aligned}
$$

where $n_{d}, n_{c} \in \mathbb{Z}_{+}, q=\max \left(n_{d}, n_{c}\right), \mathbf{X}_{i}(t), \mathbf{Y}_{i}(t) \in \mathcal{F}_{2}^{q}$ for $i=1,2$, and $\mathbf{S}$ is a $q \times q$ shift matrix. The deterministic $X$ channel is physically the same channel as the deterministic interference channel introduced in [6], except that the $X$ channel has 4 independent messages. Please refer to [6] for the illustration of the deterministic channel. The message set 
and standard definitions and notations of the achievable rates are similar to those in the Gaussian setting given in the next subsection. To avoid confusion, sometimes we add the subscript det to distinguish the notations of the deterministic channel from those for the Gaussian channel.

\section{B. The Symmetric Gaussian X Channel}

The symmetric 2-user Gaussian $X$ channel is described by the input-output equations

$$
\begin{aligned}
& Y_{1}(t)=H_{d} X_{1}(t)+H_{c} X_{2}(t)+Z_{1}(t) \\
& Y_{2}(t)=H_{c} X_{1}(t)+H_{d} X_{2}(t)+Z_{2}(t)
\end{aligned}
$$

where at symbol index $t, Y_{j}(t)$ and $Z_{j}(t)$ are the channel output symbol and additive white Gaussian noise (AWGN) respectively at receiver $j . H_{c}$ and $H_{d}$ are the channel gain coefficients, and $X_{i}(t)$ is the channel input symbol at transmitter $i$. All symbols are real and the channel coefficients do not vary with time. In the remainder of this paper, we suppress time index $t$ if no confusion would be caused. The AWGN is normalized to have zero mean and unit variance and the input power constraint is given by

$$
\mathbf{E}\left[X_{i}^{2}\right] \leq 1, \quad i=1,2 .
$$

There are 4 independent messages in the $X$ channel: $W_{11}, W_{12}, W_{21}, W_{22}$ where $W_{j i}$ represents the message from transmitter $i$ to receiver $j$. We indicate the size of the message by $\left|W_{j i}\right|$. For codewords spanning $T$ symbols, rates $R_{j i}=\frac{\log \left|W_{j i}\right|}{T}$ are achievable if the probability of error for all messages can be simultaneously made arbitrarily small by choosing an appropriate large $T$. The capacity region $\mathcal{C}$ of the $X$ channel is the set of all achievable rate tuples $\mathbf{R}=\left(R_{11}, R_{12}, R_{21}, R_{22}\right)$. We indicate the sum rate and the sum capacity of the $X$ channel by $R_{\Sigma}$ and $C_{\Sigma}$ respectively.

1) Generalized Degrees of Freedom (GDOF): The same problem formulation has been given in [7], and we include it for the sake of completeness. To motivate our problem formulation, we briefly revisit the framework for the GDOF characterization of the symmetric interference channel. The interference channel is defined as:

$$
\begin{aligned}
& Y_{1}(t)=\sqrt{\mathrm{SNR}} X_{1}(t)+\sqrt{\operatorname{INR}} X_{2}(t)+Z_{1}(t) \\
& Y_{2}(t)=\sqrt{\mathrm{INR}} X_{1}(t)+\sqrt{\mathrm{SNR}} X_{2}(t)+Z_{2}(t)
\end{aligned}
$$

and with the parameter $\alpha$ defined as follows

$$
\alpha \triangleq \frac{\log (\mathrm{INR})}{\log (\mathrm{SNR})}
$$

the GDOF metric is defined as [4],

$$
d(\alpha)=\limsup _{\mathrm{SNR} \rightarrow \infty} \frac{C_{\Sigma}(\mathrm{SNR}, \alpha)}{\frac{1}{2} \log (\mathrm{SNR})}
$$

where $C_{\Sigma}(\mathrm{SNR}, \alpha)$ is the sum capacity of the interference channel.

Since our goal is to compare GDOF of the $X$ channel with the interference channel, we use the same symmetric interference channel model described above as the physical

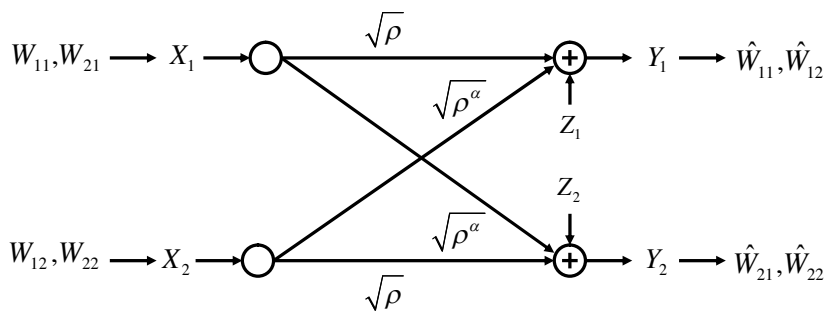

Fig. 1. 2-user Gaussian $X$ channel.

channel model for the $X$ channel. There is however, one notational difference. Since the terminology SNR, INR is not as appropriate for the $X$ channel, we instead use the parameter $\rho$ to substitute for these notions, resulting in the following system model for the $X$ channel GDOF characterization:

$$
\begin{aligned}
& Y_{1}(t)=\sqrt{\rho} X_{1}(t)+\sqrt{\rho^{\alpha}} X_{2}(t)+Z_{1}(t) \\
& Y_{2}(t)=\sqrt{\rho^{\alpha}} X_{1}(t)+\sqrt{\rho} X_{2}(t)+Z_{2}(t)
\end{aligned}
$$

In other words, we have set $H_{d}=\sqrt{\rho}, H_{c}=\sqrt{\rho^{\alpha}}$. Note that (10), (11) represent the same physical channel as (6), (7). However, as mentioned earlier, unlike the interference channel the $X$ channel has 4 independent messages: one from each transmitter to each receiver. The GDOF characterization for the $X$ channel is defined as:

$$
d(\alpha)=\limsup _{\rho \rightarrow \infty} \frac{C_{\Sigma}(\rho, \alpha)}{\frac{1}{2} \log (\rho)}
$$

where $C_{\Sigma}(\rho, \alpha)$ is the sum capacity of the $X$ channel.

Note that we use limsup to ensure that $d(\alpha)$ always exits. The half in the denominator is because all signals and channel gains are real.

\section{Main Results}

The first main result of the paper is the characterization of the sum capacity of the symmetric deterministic $X$ channel. The result is given in the following theorem.

Theorem 1: The sum capacity $C_{\Sigma}\left(n_{c}, n_{d}\right)$ of the symmetric deterministic $X$ channel is given as follows.

$$
C_{\Sigma}\left(n_{c}, n_{d}\right)= \begin{cases}2 n_{d}-2 n_{c}, & 0 \leq \frac{n_{c}}{n_{d}}<1 / 2 \\ 2 n_{c}, & 1 / 2 \leq \frac{n_{c}}{n_{d}}<3 / 4 \\ 2\left(n_{d}-\frac{1}{3} n_{c}\right), & 3 / 4 \leq \frac{n_{c}}{n_{d}}<1 \\ n_{d}, & n_{c}=n_{d} \\ 2\left(n_{c}-\frac{1}{3} n_{d}\right), & 1<\frac{n_{c}}{n_{d}} \leq 4 / 3 \\ 2 n_{d}, & 4 / 3<\frac{n_{c}}{n_{d}} \leq 2 \\ 2 n_{c}-2 n_{d}, & \frac{n_{c}}{n_{d}}>2\end{cases}
$$

The key features of the sum-capacity-achieving scheme for the regimes of $3 / 4 \leq \frac{n_{c}}{n_{d}}<1$ and $1<\frac{n_{c}}{n_{d}} \leq 4 / 3$ are shown in Fig. 2 . It can be easily seen that all interference is aligned and that all intended messages can be recovered after canceling both the decoded messages and the decoded sums of the aligned interference. 


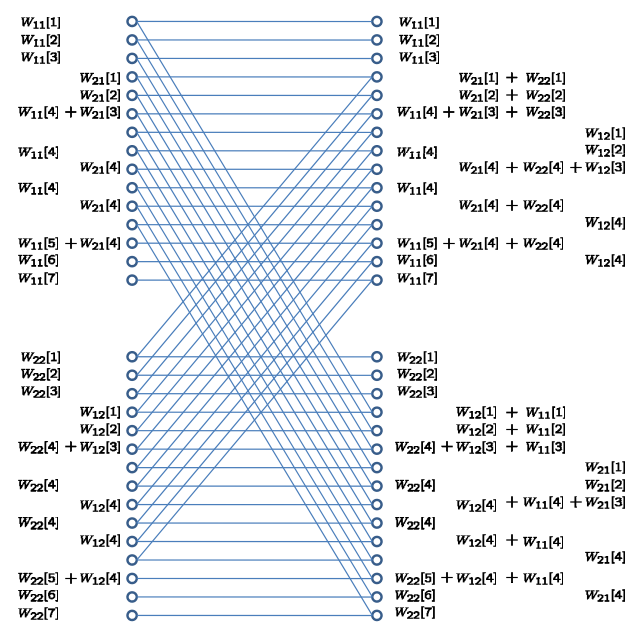

Fig. 2. Sum-capacity-achieving scheme for the symmetric deterministic $X$ channel with $\left(n_{c}, n_{d}\right)=(12,15)$

The second main result of the paper builds upon the result of Theorem 1 to find the GDOF characterization of the Gaussian $X$ channel.

Theorem 2: The generalized degrees of freedom $d(\alpha)$ of the symmetric Gaussian $X$ channel is shown in Fig. 3 and given as follows.

$$
d(\alpha)= \begin{cases}2-2 \alpha, & 0 \leq \alpha<1 / 2 \\ 2 \alpha, & 1 / 2 \leq \alpha<3 / 4 \\ 2-\frac{2}{3} \alpha, & 3 / 4 \leq \alpha<1 \\ 1, & \alpha=1 \\ 2 \alpha-\frac{2}{3}, & 1<\alpha \leq 4 / 3 \\ 2, & 4 / 3<\alpha \leq 2 \\ 2 \alpha-2, & \alpha>2\end{cases}
$$

It can be easily seen that the $X$ channel outperforms the underlying interference channel in the regimes of $2 / 3 \leq \alpha<1$ and $1<\alpha \leq 3 / 2$.

\section{Sum Capacity of the Symmetric Deterministic CHANNEL}

The goal of this section is to prove Theorem 1. We start from the following lemma, which follows trivially from the symmetry in the $X$ channel.

Lemma 3: $C_{\Sigma}\left(n_{c}, n_{d}\right)=C_{\Sigma}\left(n_{d}, n_{c}\right)$.

\section{A. Upperbound}

The following lemma provides a set of outer bounds for all achievable rate tuples $\mathbf{R}$ of the symmetric deterministic $X$ channel.

Theorem 4: All achievable rate tuples $\left(R_{11}, R_{21}, R_{12}, R_{22}\right)$ satisfy

$$
\begin{aligned}
& R_{11}+R_{12}+R_{22} \leq \max \left(n_{d}, n_{c}\right)+\left(n_{d}-n_{c}\right)^{+} \\
& R_{11}+R_{21}+R_{22} \leq \max \left(n_{c}, n_{d}\right)+\left(n_{d}-n_{c}\right)^{+} \\
& R_{11}+R_{21}+R_{12} \leq \max \left(n_{d}, n_{c}\right)+\left(n_{c}-n_{d}\right)^{+} \\
& R_{21}+R_{12}+R_{22} \leq \max \left(n_{c}, n_{d}\right)+\left(n_{c}-n_{d}\right)^{+}
\end{aligned}
$$

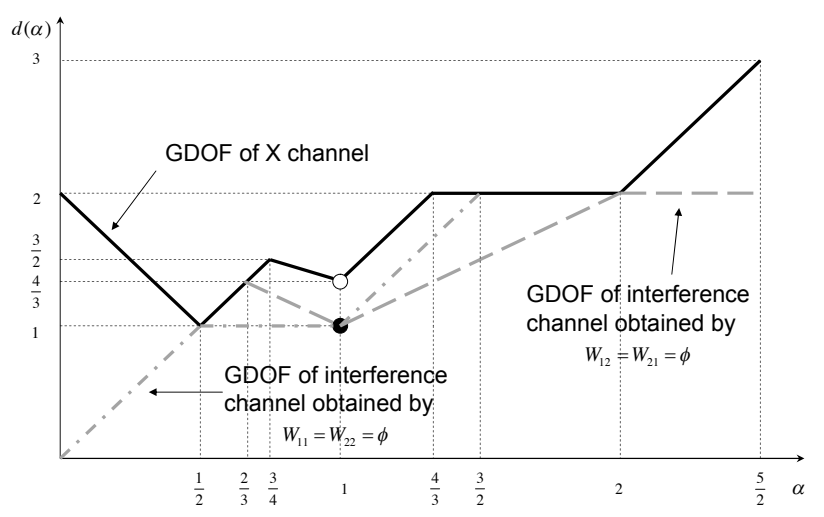

Fig. 3. Generalized degrees of freedom of the 2-user Gaussian $X$ channel.

$$
\begin{aligned}
& R_{\Sigma} \leq 2 \max \left(n_{c},\left(n_{d}-n_{c}\right)^{+}\right) \\
& R_{\Sigma} \leq 2 \max \left(n_{d},\left(n_{c}-n_{d}\right)^{+}\right)
\end{aligned}
$$

Proof: Inequality (15) to (18) are the upperbounds of the sum capacity for the four $Z$ channels contained in the $X$ channel respectively. Inequality (19) and (20) can be seen as the $X$ channel extensions of the bound given in (9.c) of [8]. Please see the full paper [9] for the detailed proof in a more general asymmetric setting.

It is easy to verify that (19) and (20) lead to the tight upperbounds of the sum capacity in the regimes of $0 \leq \frac{n_{c}}{n_{d}}<3 / 4$ and $\frac{n_{c}}{n_{d}}>4 / 3$ respectively. Adding (15) to (18) and dividing both sides by 3 , we have

$$
R_{\Sigma} \leq \frac{4}{3} \max \left(n_{c}, n_{d}\right)+\frac{2}{3}\left(n_{d}-n_{c}\right)^{+}+\frac{2}{3}\left(n_{c}-n_{d}\right)^{+} .
$$

The tight upperboud of the sum capacity for $\frac{3}{4} \leq \frac{n_{c}}{n_{d}} \leq \frac{4}{3}$ follows from (21) and the multiple access bound that if $n_{c}=$ $n_{d}$, we have $R_{\Sigma} \leq n_{d}$.

\section{B. Achievable Scheme}

The achievable scheme for $\frac{n_{c}}{n_{d}}<\frac{2}{3}$ and $\frac{n_{c}}{n_{d}}>\frac{3}{2}$ follows trivially from [6]. We provide an outline of the achievability proof for $\frac{2}{3} \leq \frac{n_{c}}{n_{d}} \leq \frac{3}{2}$. We start from the following lemma.

Lemma 5: Let $\left(n_{c}, n_{d}\right) \in \mathbb{Z}_{+}^{2}$ such that $\frac{3}{4} \leq \frac{n_{c}}{n_{d}}<1$. Then

1) If $n_{c}$ is divisible by 3 , then there exists a $\mathbf{V} \in \mathcal{F}_{2}^{n_{d} \times \frac{n_{c}}{3}}$ such that

$$
\operatorname{rank}\left(\left[\begin{array}{llll}
\mathbf{V} & \mathbf{S}^{n_{d}-n_{c}} \mathbf{V} & \mathbf{S}^{2 n_{d}-2 n_{c}} \mathbf{V} & \mathbf{V}_{\text {null }}
\end{array}\right]\right)=n_{d}
$$

where $\mathbf{V}_{\text {null }}$ is a $n_{d} \times\left(n_{d}-n_{c}\right)$ matrix whose column vectors form a basis for the nullspace of $\mathbf{S}^{n_{d}-n_{c}}$

2) There exists a $\overline{\mathbf{V}} \in \mathcal{F}_{2}^{3 n_{d} \times n_{c}}$ such that

$$
\operatorname{rank}\left(\left[\begin{array}{llll}
\overline{\mathbf{V}} & \overline{\mathbf{H}} \overline{\mathbf{V}} & \overline{\mathbf{H}}^{2} \overline{\mathbf{V}} & \overline{\mathbf{V}}_{\text {null }}
\end{array}\right]\right)=3 n_{d}
$$

where

$$
\overline{\mathbf{H}}=\left[\begin{array}{lll}
\mathbf{S}^{n_{d}-n_{c}} & \mathbf{0}_{n_{d} \times n_{d}} & \mathbf{0}_{n_{d} \times n_{d}} \\
\mathbf{0}_{n_{d} \times n_{d}} & \mathbf{S}^{n_{d}-n_{c}} & \mathbf{0}_{n_{d} \times n_{d}} \\
\mathbf{0}_{n_{d} \times n_{d}} & \mathbf{0}_{n_{d} \times n_{d}} & \mathbf{S}^{n_{d}-n_{c}}
\end{array}\right]
$$




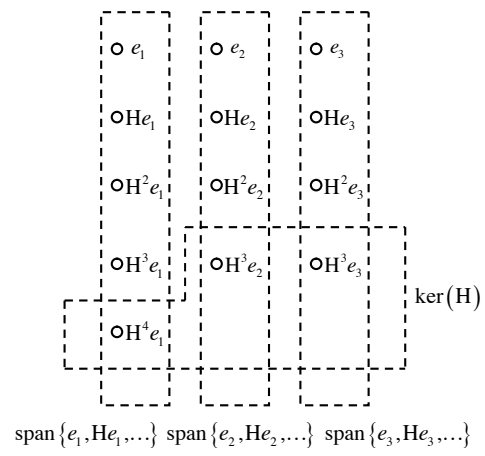

Fig. 4. A pictorial representation of the cyclic decomposition of $\mathcal{F}_{2}^{n_{d}}$ with $\left(n_{c}, n_{d}\right)=(10,13) . \mathbf{H}=\mathbf{S}^{n_{d}-n_{c}}$

and $\overline{\mathbf{V}}_{\text {null }}$ represents the $3 n_{d} \times\left(3 n_{d}-3 n_{c}\right)$ matrix whose column vectors form a basis for the nullspace of $\overline{\mathbf{H}}$.

Proof: To prove the lemma, we make use of cyclic decomposition, the idea of decomposing $\mathcal{F}_{2}^{n_{d}}$ into several disjoint invariant subspaces. Fig. 4 gives an illustration of the cyclic decomposition. Pleas see [9] for the detailed proof.

By Lemma 3, we only need to consider the achievable scheme for $n_{c} \leq n_{d}$. The achievable scheme is split into three different regimes viz. $\frac{2}{3} \leq \frac{n_{c}}{n_{d}}<\frac{3}{4}, \frac{3}{4} \leq \frac{n_{c}}{n_{d}}<1$, and $\frac{n_{c}}{n_{d}}=1$.

Achievability for $\frac{n_{c}}{n_{d}}=1$ is trivial, since an optimal achievable scheme sets $W_{12}=W_{21}=W_{22}=\phi$ and uses all the $n_{d}$ levels for $W_{11}$ at transmitter 1 . We will treat the other 2 cases below.

Case $1: \frac{2}{3} \leq \frac{n_{c}}{n_{d}}<\frac{3}{4}$

The achievable scheme for this regime is illustrated in Fig. 5 .

Case $2: \frac{3}{4} \leq \frac{n_{c}}{n_{d}}<1$

We first consider the case where $n_{c}$ is a multiple of 3 .

1) Transmit Scheme: We use linear precoding at the transmitters. Let $\mathbf{V}_{\text {null }} \in \mathcal{F}_{2}^{n_{d} \times\left(n_{d}-n_{c}\right)}$ satisfy

$$
\mathbf{S}^{n_{d}-n_{c}} \mathbf{V}_{\text {null }}=\mathbf{0}_{n_{d} \times\left(n_{d}-n_{c}\right)} .
$$

At transmitter $i$, we use, as precoding vectors for $W_{i i}$, column vectors of the matrix $\left[\begin{array}{ll}\mathbf{V} & \mathbf{V}_{\text {null }}\end{array}\right]$ where $\mathbf{V} \in \mathcal{F}_{2}^{n_{d} \times \frac{n_{c}}{3}}$. We will shortly explain how $\mathbf{V}$ is chosen, but here we mention that the columns of $\mathbf{V}$ are linearly independent of $\mathbf{V}_{\text {null }}$. Note that this implies that $\mathbf{S}^{n_{d}-n_{c}} \mathbf{V}$ has a full rank of $n_{c} / 3$. For $W_{j i}$, we use $\mathbf{S}^{n_{d}-n_{c}} \mathbf{V}$ as the precoding matrix so that the transmitted codeword $\mathbf{X}_{i}$ can be represented as

$$
\mathbf{X}_{i}=\mathbf{V} \hat{\mathbf{X}}_{i i}(1)+\mathbf{V}_{\text {null }} \hat{\mathbf{X}}_{i i}(2)+\mathbf{S}^{n_{d}-n_{c}} \mathbf{V} \hat{\mathbf{X}}_{j i}
$$

for $(i, j) \in\{(1,2),(2,1)\}$, where $\hat{\mathbf{X}}_{i i}(1) \in \mathcal{F}_{2}^{\frac{n_{c}}{3}}$ and $\hat{\mathbf{X}}_{i i}(2) \in \mathcal{F}_{2}^{n_{d}-n_{c}}$ are column vectors representing the bits encoding $W_{i i}$. $\hat{\mathbf{X}}_{j i} \in \mathcal{F}_{2}^{\frac{n_{c}}{3}}$ is the column vector of the bits encoding $W_{j i}$.

2) Receive Scheme: Consider receiver 1. The received signal $\mathbf{Y}_{1}$ can be expressed as follows.

$$
\begin{aligned}
& \mathbf{V} \hat{\mathbf{X}}_{11}(1)+\mathbf{V}_{\text {null }} \hat{\mathbf{X}}_{11}(2)+\mathbf{S}^{n_{d}-n_{c}} \mathbf{V}\left(\hat{\mathbf{X}}_{21}+\hat{\mathbf{X}}_{22}(1)\right) \\
& +\mathbf{S}^{2 n_{d}-2 n_{c}} \mathbf{V} \hat{\mathbf{X}}_{12}
\end{aligned}
$$

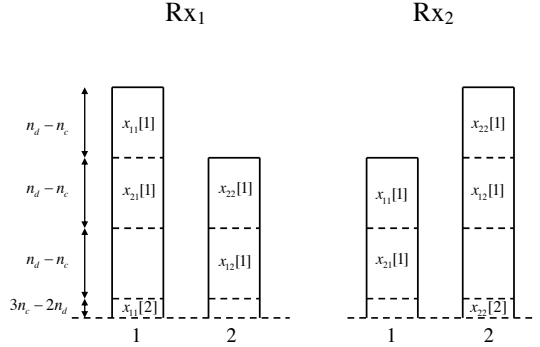

Fig. 5. Signal levels at receivers for $\frac{2}{3} \leq \frac{n_{c}}{n_{d}}<\frac{3}{4}$.

Now, receiver 1 wishes to decode $\hat{\mathbf{X}}_{11}(1), \hat{\mathbf{X}}_{11}(2), \hat{\mathbf{X}}_{12}$ using linear decoding. Notice that the interference from $\hat{\mathbf{X}}_{21}, \hat{\mathbf{X}}_{22}(1)$ aligns along $\mathbf{S}^{n_{d}-n_{c}} \mathbf{V}$. Suppose we choose $\mathbf{V}$ such that the columns of the matrix

$$
\mathbf{G}=\left[\begin{array}{lllll}
\mathbf{V} & \mathbf{S}^{n_{d}-n_{c}} \mathbf{V} & \mathbf{S}^{2 n_{d}-2 n_{c}} \mathbf{V} & \mathbf{V}_{\text {null }}
\end{array}\right]
$$

are linearly independent, then clearly receiver 1 can decode $W_{11}, W_{12}$ using linear decoding. Therefore, in order to show achievability, we need to show that there exists $\mathbf{V}$ so that the matrix $\mathbf{G}$ has a full rank of $n_{d}$. This is shown in Lemma 5 . Similar analysis applies to receiver 2 .

Now, we consider the case where $n_{c} / 3$ is not an integer. In this case, we use a 3 symbol extension of the channel represented below

$$
\underbrace{\left[\begin{array}{l}
\mathbf{Y}_{i}(3 t) \\
\mathbf{Y}_{i}(3 t+1) \\
\mathbf{Y}_{i}(3 t+2)
\end{array}\right]}_{\overline{\mathbf{Y}}_{i} \in \mathcal{F}_{2}^{3 n_{d}}}=\underbrace{\left[\begin{array}{l}
\mathbf{X}_{i}(3 t) \\
\mathbf{X}_{i}(3 t+1) \\
\mathbf{X}_{i}(3 t+2)
\end{array}\right]}_{\overline{\mathbf{X}}_{i} \in \mathcal{F}_{2}^{3 n_{d}}}+\overline{\mathbf{H}} \underbrace{\left[\begin{array}{l}
\mathbf{X}_{j}(3 t) \\
\mathbf{X}_{j}(3 t+1) \\
\mathbf{X}_{j}(3 t+2)
\end{array}\right]}_{\overline{\mathbf{X}}_{j} \in \mathcal{F}_{2}^{3 n_{d}}}
$$

Like the case where $n_{c}$ was a multiple of 3 , a linear precoding and decoding technique is applicable over this extended channel. The only difference in this case is that, we need to show that there exists a $3 n_{d} \times n_{c}$ matrix $\overline{\mathbf{V}}$ such that the matrix

$$
\overline{\mathbf{G}}=\left[\begin{array}{llll}
\overline{\mathbf{V}} & \overline{\mathbf{H}} \overline{\mathbf{V}} & \overline{\mathbf{H}}^{2} \overline{\mathbf{V}} & \overline{\mathbf{V}}_{\text {null }}
\end{array}\right]
$$

has a full rank of $3 n_{d}$, where $\overline{\mathbf{V}}_{\text {null }}$ represents the $\left(3 n_{d}-3 n_{c}\right)$ basis elements of the null space of $\overline{\mathbf{H}}$. This is shown in Lemma 5 as well. This completes the proof of the achievability.

Remark: In the regime of $\frac{3}{4} \leq \frac{n_{c}}{n_{d}}<1$, the sum-capacityachieving scheme needs to satisfy the challenging condition of achieving (15) to (18) simultaneously. And the scheme solves the problem by efficiently aligning the interference: for the $n_{c}$ levels that receiver $j$ can see the signals from both transmitters, $\frac{n_{c}}{3}$ of them are used for $W_{j j}, \frac{n_{c}}{3}$ of them are used for $W_{j i}$, and $\frac{n_{c}}{3}$ of them are used for aligning $W_{i i}$ and $W_{i j}, i \neq j$.

\section{Generalized Degrees of Freedom of the SYMMETRIC GAUSSIAN $X$ CHANNEL}

To prove Theorem 2, we start from the following lemma. Lemma 6: $d(\alpha)=\alpha d\left(\frac{1}{\alpha}\right)$.

Proof: The lemma is proved using the symmetry of the $X$ channel. Please see [9] for the details. 
By Lemma 6 , we only need to find $d(\alpha)$ for $\alpha \leq 1$. Since $d(\alpha)$ for $\alpha \leq \frac{2}{3}$ has been established in Theorem 2 of [7], we only consider the remaining case of $\frac{2}{3}<\alpha \leq 1$.

The upperbounds are derived using the insights obtained from the deterministic setting. Please see [9] for the detailed derivation and the connection between the deterministic and Gaussian cases.

The achievable scheme is similar to those used in [10], [11], and we include an outline of the proof for the sake of the completeness.

For a given $\alpha \in\left[\frac{2}{3}, 1\right]$, we can find a $\left(n_{d}, n_{c}\right) \in \mathbb{Z}_{+}^{2}$ and a very small nonnegative value $\epsilon$ such that

$$
\alpha=\frac{1}{n_{d}}\left(n_{c}+\epsilon\left(n_{d}-n_{c}\right)\right) .
$$

Note that when $\alpha$ is a rational number, $\epsilon$ is chosen to be zero. But when $\alpha$ is not rational, $\epsilon\left(n_{d}-n_{c}\right) / n_{d}$ is used to compensate the difference between $\alpha$ and a rational number $\frac{n_{c}}{n_{d}}$ that is very close to $\alpha$. We choose $\left(n_{c}, n_{d}\right)$ such that (14) can be achieved without symbol extension for the symmetric deterministic channel with parameter $\left(n_{c}, n_{d}\right)$.

Consider the sequence of channels, i.e. $\rho$ indexed by $N$, such that

$$
\rho=Q^{\frac{2 N n_{d}}{1-\epsilon}}
$$

where $Q$ is a very large but fixed positive integer and $N$ is a positive integer whose value grows to infinity. Note that $\rho$ grows to infinity as $N$ grows to infinity.

1) Transmit Scheme: We impose the following structure on the Q-ary representation of the transmit signal $X_{i}$ at transmitter $i$ for $i \in\{1,2\}$.

$$
X_{i}=\frac{1}{\sqrt{\rho}} \sum_{k=0}^{N n_{d}-1} x_{i, k} Q^{k}
$$

The values of $x_{i, k}$ are restricted to the set $\left\{1, \ldots,\left\lfloor\frac{Q-1}{4}-1\right\rfloor\right\}$ to ensure that the addition of interference does not produce carry over. It's easy to see that the power constraint is satisfied.

Since the signal design process developed in the Section IV-B also works in $\mathcal{F}_{\left\lfloor\frac{Q-1}{4}\right\rfloor-2}^{N n_{d}}$, we can use it to find the transmit signals $\mathbf{X}_{1}, \mathbf{X}_{2} \in \mathcal{F}_{\left\lfloor\frac{Q-1}{4}\right\rfloor-2}^{N n_{d}}$ for the symmetric deterministic channel with parameter $\left(N n_{c}, N n_{d}\right)$ and then obtain the corresponding $X_{1}, X_{2} \in \mathbb{R}_{+}$by

$$
X_{i}=\frac{1}{\sqrt{\rho}}\left[\begin{array}{llllll}
Q^{N n_{d}-1} & Q^{N n_{d}-2} & \cdots & Q^{2} & Q^{1} & 1
\end{array}\right] \mathbf{X}_{i} .
$$

2) Receive Scheme : Each receiver takes the magnitude of the received signal, reduces to modulo $Q^{N n_{d}}$, discards the value below the decimal point, and expresses the result in Qary representation as

$$
\begin{aligned}
\bar{Y}_{i} & =\left\lfloor\left|Y_{i}\right| \bmod Q^{N n_{d}}\right\rfloor \\
& =\sum_{k=0}^{N n_{d}-1} y_{i, k} Q^{k}, \quad y_{i, k} \in\{0,1, \ldots, Q-1\}
\end{aligned}
$$

Substituting (24) and (25) into (10) and (11), we can rewrite the input output equation as

$$
Y_{i}=\bar{X}_{i}+Q^{N\left(n_{c}-n_{d}\right)} \bar{X}_{j}+Z_{i}, \quad(i, j) \in\{(1,2),(2,1)\}
$$

where

$$
\bar{X}_{i} \triangleq \sqrt{\rho} X_{i}
$$

Note that multiplication by $Q^{N\left(n_{c}-n_{d}\right)}$ shifts the decimal point in the Q-ary representation of $\bar{X}_{j}$ by $N\left(n_{d}-n_{c}\right)$ places to the left. Thus, in the absence of noise, the $N n_{d}$ digits of $\bar{X}_{1}, \bar{X}_{2}, \bar{Y}_{1}$, and $\bar{Y}_{2}$ behave exactly like the symmetric deterministic channel with parameter $\left(N n_{c}, N n_{d}\right)$. Following the similar arguments in [10], [11], we have

$$
\begin{aligned}
R_{\Sigma} & =R_{\Sigma, \text { det }}\left(N n_{c}, N n_{d}\right) \log _{Q}\left(\left\lfloor\frac{Q-1}{4}-2\right\rfloor\right)+o(N) \\
& =N R_{\Sigma, \text { det }}\left(n_{c}, n_{d}\right) \log _{Q}\left(\left\lfloor\frac{Q-1}{4}-2\right\rfloor\right)+o(N)
\end{aligned}
$$

Combining (25), (30), and (9), we can show that $d(\alpha)$ is not less than

$$
\begin{aligned}
& \limsup _{N \rightarrow \infty} \frac{N R_{\Sigma, \operatorname{det}}\left(n_{d}\left(\frac{\alpha-\epsilon}{1-\epsilon}\right), n_{d}\right) \log _{Q}\left(\left\lfloor\frac{Q-1}{4}-2\right\rfloor\right)+o(N)}{\frac{N n_{d}}{1-\epsilon}} \\
& =\frac{1-\epsilon}{n_{d}} R_{\Sigma, \operatorname{det}}\left(n_{d}\left(\frac{\alpha-\epsilon}{1-\epsilon}\right), n_{d}\right) \log _{Q}\left(\left\lfloor\frac{Q-1}{4}-2\right\rfloor\right)
\end{aligned}
$$

Carrying out the substitution of $R_{\Sigma, d e t}(\cdot, \cdot)$, choosing $Q$ and $\epsilon$ to be arbitrarily large and small respectively, and comparing with the outerbound, we finish the proof of Theorem 2.

\section{ACKNOWLEDGMENT}

This work was supported by NSF under Grant CCF0830809 and by ONR YIP under Grant N00014-08-1-0872.

\section{REFERENCES}

[1] S. Jafar and S. Shamai, "Degrees of freedom region for the MIMO X channel," IEEE Trans. Inform. Theory, vol. 54, pp. 151-170, Jan. 2008.

[2] M. Maddah-Ali, A. Motahari, and A. Khandani, "Communication over MIMO X channels: interference alignment, decomposition, and performance analysis," IEEE Trans. Inform. Theory, vol. 54, pp. 3457-3470, Aug. 2008.

[3] T. Philosof and R. Zamir, "The rate loss of single-letter characterization: the dirty multiple access channel," arxiv:0803.1120v3 [cs.IT], Mar. 2008.

[4] R. Etkin, D. Tse, and H. Wang, "Gaussian interference channel capacity to within one bit," IEEE Trans. Inform. Theory, vol. 54, pp. $5534-$ 5562, Dec. 2008.

[5] A. Avestimehr, S. Diggavi, and D. Tse, "A deterministic approach to wireless relay networks," arXiv:0710.3777 [cs.IT], Oct. 2008.

[6] G. Bresler and D. Tse, "The two-user Gaussian interference channel: a deterministic view," European Trans. in Telecommunications, vol. 19, pp. 333-354, June 2008.

[7] C. Huang, V. Cadambe, and S. Jafar, "Generalized degrees of freedom of the (noisy) X channel," in Proc. Asilomar Conference on Signals, Systems, and Control, Oct. 2008.

[8] A. E. Gamal and M. Costa, "The capacity region of a class of deterministic interference channels," IEEE Trans. Inform. Theory, vol. 2, pp. 343-346, March 1982.

[9] C. Huang, V. Cadambe, and S. Jafar, "On the capacity and the generalized degrees of freedom of the X channel," arXiv:0810.4741 [cs.IT], Oct. 2008

[10] S. Jafar and S. Vishwanath, "Generalized degrees of freedom of the symmetric Gaussian k user interference channel." arXiv:0804.4489 [cs.IT], Apr. 2008.

[11] V. Cadambe, S. Jafar, and S. Shamai, "Interference alignment on the deterministic channel and application to gaussian networks," IEEE Trans. Inform. Theory, vol. 55, pp. 269 - 274, Jan. 2009. 